\title{
Face Liveness Detection using Local Diffused Patterns
}

\author{
Gautam Pallavi \\ Department of Computer Science \& Engineering \\ Hindustan Institute of Technology\& Management \\ Agra, India
}

\author{
Jayash Kumar Sharma \\ Department of Computer Science \& Engineering \\ Hindustan Institute of Technology\& Management \\ Agra, India
}

\begin{abstract}
In general, face spoofing attacks deals with printing artifacts, electronic screens and ultra-realistic face masks or models. This paper proposes a liveness detection method based on diffusion speed. Diffusion speed of a single image is calculated as the difference of the original images and diffused images at each pixel. Face spoofing method based on diffusion speed does not require any user involvement and works with a single image. The key aspect of the proposed method is based on the difference in the illumination characteristics of live and fake faces. To solve the nonlinear, scalar valued diffusion equation, AOS (Additive Operator Splitting) approach, together with TDMA (Tri-Diagonal Matrix Algorithm) is applied. The local pattern of the diffusion speed is calculated at each pixel position (Local Diffused Patterns) and fed to linear Support Vector Machine for classification. Proposed approach performs well against the diverse malicious attacks, face display media (screen / paper) \& varying illuminations and gives $90.83 \%$ accuracy.
\end{abstract}

\section{General Terms}

Digital Image Processing, Pattern Recognition

\section{Keywords}

Spoofing, diffusion speed, local diffused pattern, face liveness detection, Additive Operator Splitting, Tri-Diagonal Matrix Algorithm

\section{INTRODUCTION}

In the era of information technology, there is high demand of high level security. Biometric authentication techniques have gained the considerable attention to guard against such attack. Fingerprint recognition, hand geometry, retinal and iris scanner are some of the well-known biometric techniques used for security purpose. No matter, how efficient the approaches are, these techniques require the intentional interaction with the biometric device. As an alternative, face recognition technology has gained more popularity in the recent years. Face recognition methods are direct, user friendly and convenient as compared to other biometric methods. Therefore, face recognition systems have been applied to various security systems. With the advancement of technology, face recognition systems have faced spoofing attacks made by non-real faces. A spoofing attack tries to spoof face recognition system by providing fake facial pictures through photographs, mobile phone pictures or video frames. In case of such attacks, many face recognition systems may not able to differentiate between original ('live') face and fake ('not live') face. Such spoofing attacks are major security threat to face recognition systems.

In order to protect against such spoofing attacks, a face recognition system / security system must be equipped with liveness detection. Liveness detection differentiates between the feature space of live entities (Real faces) and non-living entities (Fake faces). Attackers will try to break face recognition system by producing a large number of spoofed face inputs. Liveness detection with face recognition systems can identify such fake inputs and can help in improving the performance of biometric system.

Figure-1 represents a block diagram of a traditional liveness detection system. It accepts an input from the / sensing device, detects \& preprocesses detected face, applies liveness detection algorithms and determines whether the input image belongs to actual live user or an imposture user.

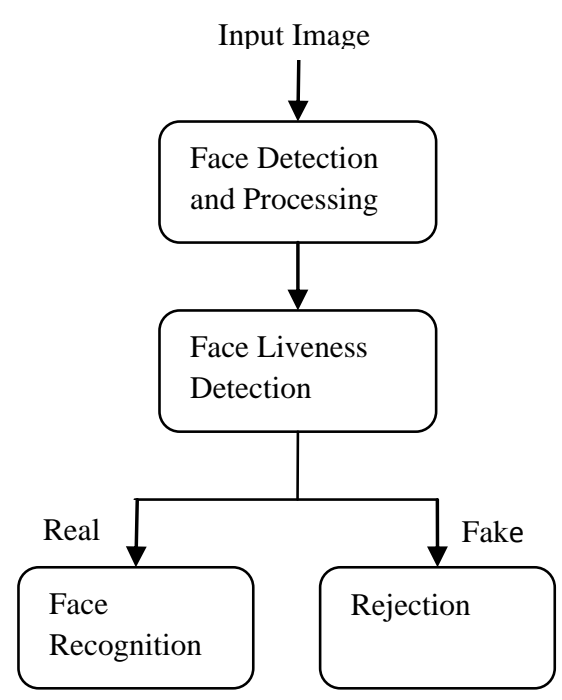

Figure-1: Liveness detection in Face recognition system

During the recent years, liveness detection has been identified as a challenging and crucial security issue which deals with the trustworthiness of biometric system security against spoofing attacks, Therefore, Liveness detection or antispoofing in biometrics systems has achieved great progress in the recent years.

\section{RELATED WORK}

Several researchers have contributed their efforts towards liveness detection in the form of texture based techniques and motion based techniques.

Texture based techniques are relatively easy to use as they work on a single image and does not require anti-spoofing databases. Such techniques aim to differentiate the fake and live using micro texture present in the printed paper.

The physics-based method [1] detects works with a single image recaptured from printed material. When light incident on image surface, some amount of light is reflected off by the surface. This reflection is referred as the specular component of image which is being used for differentiating live and recaptured / fake faces. 
Tan et. al. [2] discussed a real-time and non-intrusive Lambertian reflectance model which differentiates 2D image captured from the printed paper and a real $3 \mathrm{D}$ face using a generic web camera.

Face spoofing detection based on micro-texture analysis [3] detects whether a print image is produced in front of the camera or a live person is present. The key idea behind this approach is that printing quality defects are present in the face prints on paper which can be are easily detected using microtexture patterns. These approaches have considered a human face as a non-rigid, complex $3 \mathrm{D}$ object and a photograph as a 2D planar object. Therefore; there both objects (human faces and photograph prints) reflect light in different ways which result in different specular reflections and shades.

Motion-based approaches are considered as most suitable methods for anti-spoofing. Motion-based approaches detect various frequently occurring natural responses of the face such as Eye-blinks [4] [5] (Human eye blinks every 3-4 seconds). Scene context matching method [6] works on video and detects video-spoofing in stationary face-recognition systems. Bao et. al. [7] discussed method to analyze the facial motion using Optical Flow (OF) which differentiate between 3D objects (real face) and 2D planes (printed photograph). Optical flow can also be used to evaluate the trajectory of several face parts in a non-intrusive manner [8]. Trajectory Analysis of certain parts of live faces provides valuable information which help in discriminating live face against a spoofed face. Anjos et. al. [9] presents foreground / background motion correlation using Optical Flow (OF) to indicate the occurrence of a spoofing attack.

Santosh et al. [10] proposed the classification pipeline consisting of the recently developed data driven approach called Dynamic Mode Decomposition (DMD), involving a texture based descriptor using Local Binary Patterns and Support Vector Machines with histogram intersection kernels. Raghavendra et. al. [12] used lytro light field camera (LFC) that captures the intensities and the direction of all possible rays incident on each photo sensor pixel. Hence, in a single capture, LFC provides multiple depth images. The fake artifacts presented to the LFC will appear to represent insane objects and results in less variations of depth (or focus) as compared to the real face images which results in large variation.

Methods present in section-II are effective when used with a single input image but vulnerable to high resolution-based spoofing attacks using a large display. In order to counter this problem Kim et. al. [11] suggested diffusion based method to differentiate a live and fake face in a single image. The whole idea was based on the fact that the illumination energies diffuse slowly on a uniform 2D surface, whereas these energies move faster on a 3D live face because of its nonuniformity. Diffusion speed is calculated using Local Speed Patterns (LSPS) - local patterns of diffusion speeds and by utilizing the Total Variation flow scheme. The anti-spoofing features are extracted and fed into a linear Support Vector Machine to detect the liveness of the given face input.

\section{METHODOLOGY}

\subsection{Motivation}

The inspiration driving the proposed technique depends on the distinction in the illumination characteristics of live and fake face. When light falls on the live face, it reflects randomly in different directions due to its 3D surface (lip, nose, etc.), whereas light reflects uniformly from the planer 2D surface of the fake face such as a photograph, electronic screen. The illumination energies incident on a 2D surface are evenly distributed and hence are relied upon to diffuse gradually, though those with a live face tend to move faster on account of their non-uniformity. Figure- 2 shows illumination characteristics of fake and live face respectively.

The diffusion speed is characterized as the distinction in pixel values between the original and diffused pictures. Diffusion speed provides important data which can be utilized to separate the live face from fake face. Proposed method combines the concept of diffusion along with TDMA (TriDiagonal Matrix Algorithm).
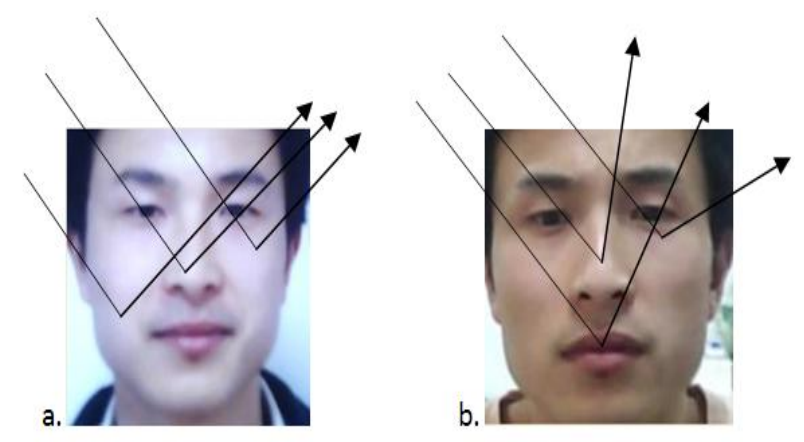

Figure-2: Illumination characteristics of (a) fake (b) live face. Sample taken from NUAA dataset

Figure-3 depicts a block diagram of the proposed methodology. Firstly, diffused image is obtained by calculating the diffusion of the original image by applying AOS (Additive Operator Splitting) scheme with TDMA (TriDiagonal Matrix Algorithm) to solve the diffusion equation. Subsequently, delta image is obtained by finding the difference of the original image and diffused image and locally diffused patterns at each pixel position of the delta image are considered as the baseline feature. Extracted features are fed to Support Vector Machine to classify whether input image is live image or fake image.

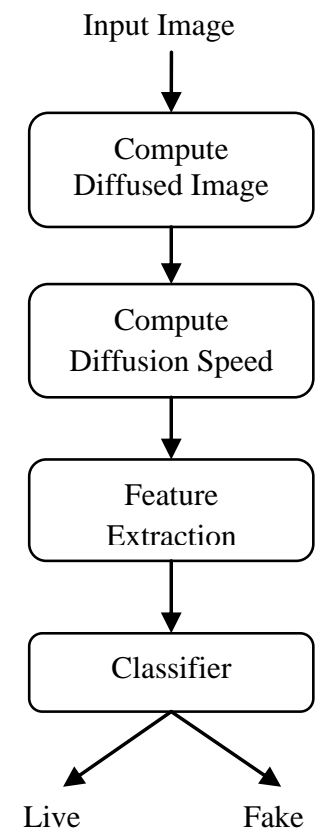

Figure-3: Overall process of the proposed system. 


\subsection{Compute Diffused Image}

In this subsection a diffused image of the input image is computed. Firstly, the nonlinear diffusion [14] is performed on input image $I$.

$u^{k+1}=u^{k}+\operatorname{div}\left(d\left(\left|\nabla u^{k}\right| \nabla u^{k}\right), u(k=0)=I\right.$

Here, $k$ denotes the iteration number.

To solve diffusion equation characterized in (1), Additive Operator Splitting (AOS) scheme [15] defined in equation-2 is used:

$$
u^{k+1}=\frac{1}{2}\left(\left(I-2 \tau A_{x}\left(u^{k}\right)\right)^{-1}+\left(I-2 \tau A_{y}\left(u^{k}\right)\right)^{-1}\right) u^{k}
$$

$A_{x}$ and $A_{y}$ represents diffusion matrices calculated in the horizontal and vertical directions, respectively. For solving AOS, we propose the use of TDMA (Tri-Diagonal Matrix Algorithm). TDMA [13] is a block solver and simplified form of Gaussian elimination which is used to solve tri-diagonal systems of equations. In matrix/vector format it can be written as:

$$
\underbrace{\left[\begin{array}{ccccc}
b_{1} & c_{1} & 0 & 0 & 0 \\
a_{2} & b_{2} & c_{2} & 0 & 0 \\
0 & a_{3} & b_{3} & \ldots & 0 \\
. & . . & . . & . . & . . \\
0 & 0 & . . & . . & c_{N-1} \\
0 & 0 & 0 & a_{N} & b_{N}
\end{array}\right]\left[\begin{array}{c}
x_{1} \\
x_{2} \\
x_{3} \\
\cdot \\
\cdot \\
x_{N}
\end{array}\right]}_{A}=\underbrace{\left[\begin{array}{c}
d_{1} \\
d_{2} \\
d_{3} \\
\cdot \\
d_{N}
\end{array}\right]}_{\mathrm{x}}
$$

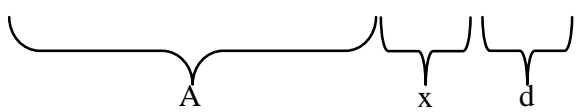

The following steps are performed by TDMA [13]:

Step-1: Forward sweep eliminates $a_{i}$ using equation-3.

$c_{i}^{\prime}= \begin{cases}\frac{c_{1}}{b_{1}} & , i=1 \\ \frac{c_{i}}{b_{i}-c_{i-1}^{\prime} a_{i}} & , i=2,3, \ldots, N-1\end{cases}$

$d_{i}^{\prime}= \begin{cases}\frac{d_{1}}{b_{1}} & , i=1 \\ \frac{d_{i}-d_{i-1}^{\prime} a_{i}}{b_{i}-c_{i-1}^{\prime} a_{i}} & , i=2,3, \ldots, N\end{cases}$

Step-2: Backward sweep computes the solution of AOS equation using equation-4.

$$
\begin{gathered}
x_{N}=d^{\prime}{ }_{N} \\
x_{i}=d_{i}^{\prime}-c^{\prime}{ }_{i} x_{i+1}, i=N-1, N-2, \ldots, 1
\end{gathered}
$$

Here, $a_{i}, b_{i}$ are the diffusion weights. In AOS, first TDMA is applied columns wise then along all the rows.

After this step, the output of the original input image is a diffused image. Figure-4(a) depicts an input image taken from NUAA dataset [2]. Diffused image obtained using AOS scheme and TDMA is shown in figure-4(b).

\subsection{Compute Diffusion Speed}

The Diffusion speed [11] is obtained by taking the difference of the diffused image and the original one at each pixel position $(x, y)$. The resulting image is being referred as Delta Image in this literature. Figure-4(c) shows a delta image or representation of diffusion speed.

\subsection{Feature Extraction Using Local Diffused Patterns}

Local Diffused Patterns (LDP) is being referred as the calculated value of diffusion speed at each pixel of the delta image. LDP is considered as the baseline features for the proposed system, which is given as [11]:

$$
F_{\text {base }}=\{s(x, y) \mid 0<x \leq \mathrm{W}, 0<y \leq \mathrm{H}\}
$$

Here, $W$ and $H$ are width and height of the identified face region, respectively.

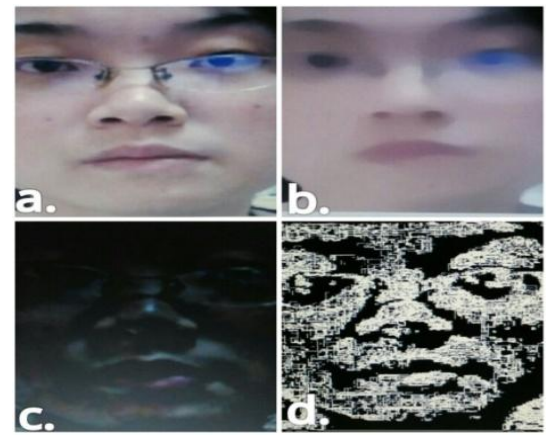

Figure-4: (a) Original image (b) Diffused Image (c) Diffusion Speed (d) Local diffused pattern

The Local Diffused Pattern (LDP) is capable enough to obtain the minute differences between diffusion speed maps of live and fake faces using equation- 6 :

$$
\begin{gathered}
F_{L D P}(x, y)=\sum_{i=1}^{n} 2^{i-1} \operatorname{LDPi}(x, y) \\
\operatorname{LDPi}=\left\{\begin{array}{l}
1, \text { if } \mathrm{s}(\mathrm{x}, \mathrm{y})>s\left(x_{i}, \mathrm{y}_{\mathrm{i}}\right) \\
0, \text { otherwise }
\end{array}\right.
\end{gathered}
$$

Here $(\mathrm{x}, \mathrm{y})$ is the center pixel, $\mathrm{n}$ is the number of neighboring pixels in the $3 \times 3$ (here $n=8)$ and $\left(x_{i}, y_{i}\right)$ indicates the position of the neighboring pixels for I ranging from 1 to 8 . Therefore; calculated $F_{\mathrm{LDP}}(\mathrm{x}, \mathrm{y})$ is in the range of $[0,255]$ as shown in figure-5. Figure-4(d) shows a gray-scale representation of the image.

\begin{tabular}{|l|l|l|}
\hline 1 & 5 & 7 \\
\hline 5 & 4 & 3 \\
\hline 8 & 2 & 0 \\
\hline
\end{tabular}$\stackrel{\text { Threshold }}{\longrightarrow}$\begin{tabular}{|c|c|c|}
\hline 0 & 1 & 1 \\
\hline 1 & & 0 \\
\hline 1 & 0 & 0 \\
\hline
\end{tabular}

Binary $=01100011$

Decimal $=99$ 


\section{EXPERIMENTAL RESULTS}

\subsection{NUAA Dataset}

In this paper, dataset provided by NUAA [2] is used. The NUAA dataset is most widely adopted dataset for face liveness detection. It consists of 15 subjects looking at the webcam with a neutral expression. Images are captured at 20 fps. For creating the fake images, pictures of the subjects have taken using the Cannon camera, and printed on the photographic paper as well as a normal A4 sheet. These printed photographs are then shown to the webcam to create the fake images. Samples taken from NUAA dataset are shown in figure-6.

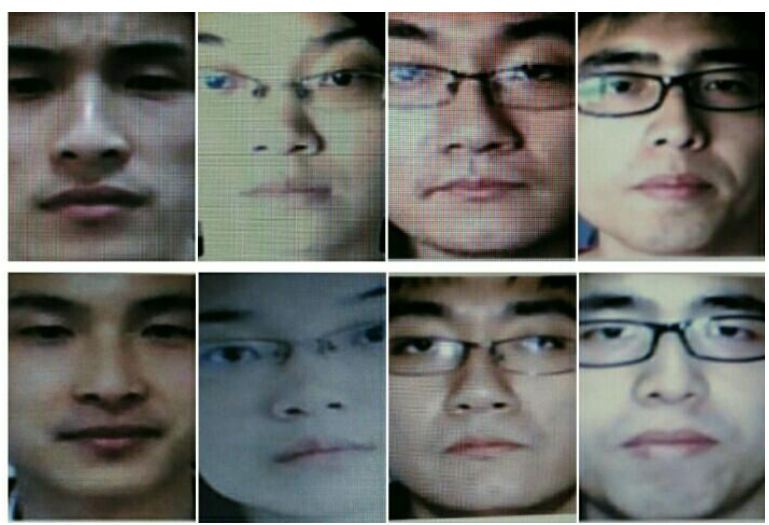

Figure-6: Sample images from NUAA dataset, real (top row) and fake (bottom row)

\subsection{Experiments and Results}

For any given image, diffused image is computed and diffusion speed is calculated. Further, feature vector based on diffusion speed is calculated and the system is trained with Support Vector Machine. Figure-7 shows the results obtained in intermediate steps. The system is trained with 60 real images and 60 fake images (total 120 images). For classification purpose, 120 random images (which include 53 real images and 67 fake images) are used. Table-1 summarizes images used for training and classification.

Table 1. Dataset Summary

\begin{tabular}{|c|c|c|c|}
\hline & Real & Fake & Total \\
\hline Training & 60 & 60 & 120 \\
\hline Testing & 53 & 67 & 120 \\
\hline
\end{tabular}

The proposed method provides $90.83 \%$ accuracy by detecting 109 images correctly out of 120 images. 47 images are correctly classified out of 53 real images, whereas 62 fake images are correctly classified out of 67 fake images with $88.67 \%$ and $92.53 \%$ accuracy respectively as shown in table2. Graphical representation of results is shown in figure-8.

Table 2. Classification Details

\begin{tabular}{|c|c|c|c|}
\hline & $\begin{array}{c}\text { Total } \\
\text { Images }\end{array}$ & $\begin{array}{c}\text { Correctly } \\
\text { Identified }\end{array}$ & Accuracy\% \\
\hline Real Images & 53 & 47 & $88.67 \%$ \\
\hline Fake Images & 67 & 62 & $92.53 \%$ \\
\hline Total & 120 & 109 & $90.88 \%$ \\
\hline
\end{tabular}

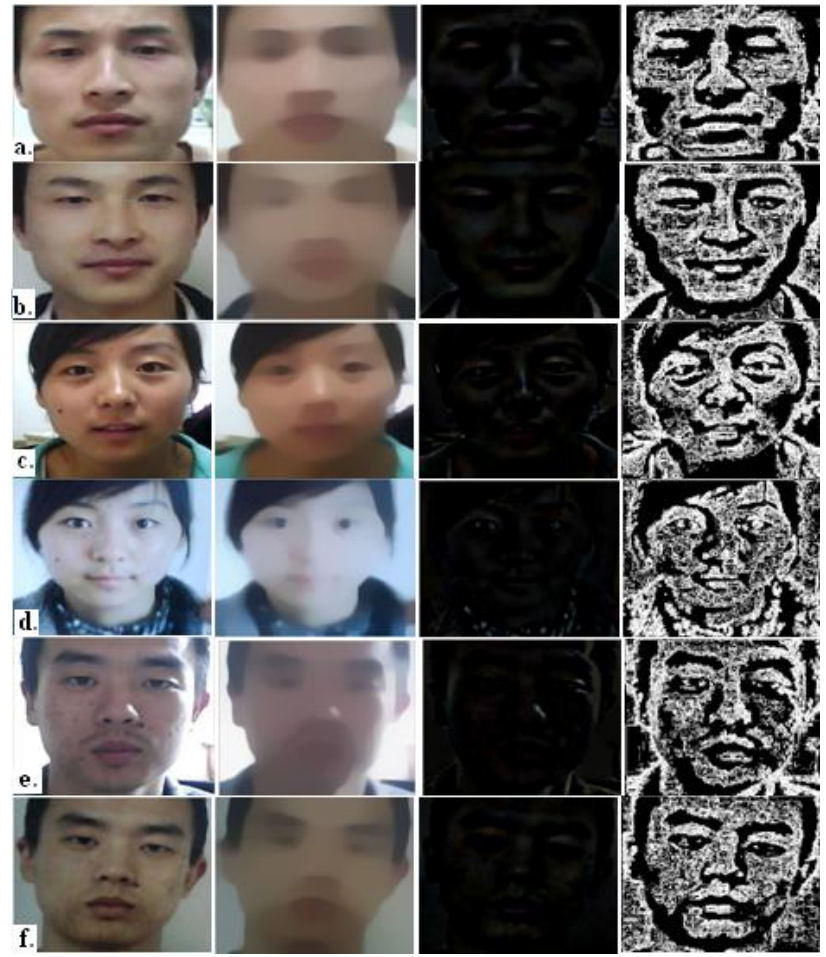

Figure-7: (a),(c),(e) are the real images and (b),(d),(f) are fake image. The first column shows the images taken from the NUAA dataset. Diffusion images, diffusion speed, LDP are shown in second, third, fourth column respectively.

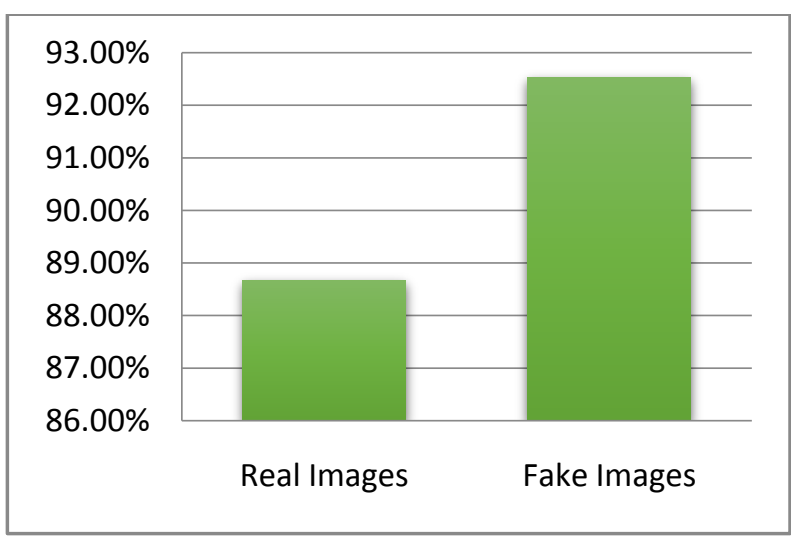

Figure-8: Classification Results

\section{CONCLUSION}

Face liveness detection method discussed in this paper uses the concept of diffusion speed to get the valuable information to differentiate the live face from the fake face by conducting nonlinear diffusion on the face image to get the diffused image. The diffusion equation is solved with the help of AOS (Additive Operator Splitting) scheme and TDMA (TriDiagonal Matrix Algorithm). Then, delta image is calculated by taking the difference of diffused image from the original image. In this method, LDP (local diffused pattern) is considered as our baseline feature which is diffused pattern at each pixel position. LDP is fed to linear Support Vector Machine for training and testing. Based on experimental result, it is observed that system gives $90.88 \%$ accuracy. 


\section{ACKNOWLEDGMENTS}

Authors are thankful to Hindustan Institute of Technology \& Management, Agra for providing necessary resources and infrastructure.

\section{REFERENCES}

[1] J. Bai, T. Ng, X. Gao, and Y. Shi, "Is physics-based liveness detection truly possible with a single image?", IEEE International Symposium on Circuits and Systems, 2010, pp. 3425-3428.

[2] X. Tan, Y. Li, J. Liu, and L. Jiang, "Face liveness detection from a single image with sparse low rank bilinear discriminative model", Computer Vision ECCV 2010, vol. 6316, pp. 504-517, 2010.

[3] J. M"a"att"a, A. Hadid, and M. Pietik"ainen, "Face spoofing detection from single image using micro-texture analysis," International Joint Conference on Biometrics (IJCB), 2011.

[4] G. Pan, L. Sun, Z. Wu, and S. Lao, "Eyeblink-based antispoofing in face recognition from a generic web camera," IEEE $11^{\text {th }}$ International Conference on Computer Vision, 2007, pp. 1-8.

[5] G. Pan, Z. Wu, and L. Sun, "Liveness detection for face recognition," Recent Advances in Face Recognition, December 2008, pp. 109-124.

[6] G. Pan, L. Sun, Z. Wu, and Y. Wang, "Monocular camera-based face liveness detection by combining eyeblink and scene context" Journal of Telecommunication Systems, 2009.

[7] W. Bao, H. Li, N. Li, and W. Jiang, "A liveness detection method for face recognition based on optical flow field," IEEE International Conference on Image Analysis and Signal Processing, 2009, pp. 233-236.
[8] K. Kollreider, H. Fronthaler, J. Bigun, "Non-intrusive liveness detection by face images" Image and Vision Computing, 2009, vol. 27, no. 3, pp. 233-244.

[9] A. Anjos, M. M. Chakka, and S. Marcel, "Motion-based counter measures to photo attacks in face recognition" IET Biometrics, vol. 3, no. 3, pp. 147-158, September 2014.

[10] Santosh Tirunagari, Norman Poh, David Windridge, Aamo Iorliam, Nick Suki, Anthony T. S. Ho, "Detection of face spoofing using visual dynamics" IEEE Transaction on Information Forensics and Security", vol. 10, No. 4, April 2015.

[11] Won Jun Kim, Sungjoo Suh, Jae Joon Han, "face liveness detection from single image via diffusion speed model" IEEE Transaction on Image Processing, vol. 24, No. 8, August 2015.

[12] R. Raghavendra, Kiran. B. Raju, Christoph Bush, "Presentation attack detection for face recognition using Light field camera", IEEE Transaction on Image Processing, vol. 24, No. 3, March 2015

[13] J. Ralli "PDE Based Image Diffusion and AOS", PhD thesis, 2014.

[14] P. Perona and J. Malik, "Scale-space and edge detection using anisotropic diffusion" IEEE Transaction on Pattern Analysis and Machine Intelligence, vol. 12, no. 7, pp. 629-639, July 1990

[15] J. Weickert, B.M.T.H. Romeny, and M.A. Viergever, "Efficient and reliable schemes for nonlinear diffusion filtering" IEEE Transaction on Image Processing, vol. 7 no. 3, pp. 398-410, Mar. 1998. 